\title{
Downlink Scheduling and Resource Management for Best Effort Service in TDD-OFDMA Cellular Networks
}

\author{
Young Min Ki, Eun Sun Kim, and Dong Ku Kim \\ Yonsei University, Dept. of Electrical and Electronic Engineering \\ 134 Shinchon Dong, Seodaemun Gu, Seoul, Korea \\ \{mellow, esunkim, dkkim\}@yonsei.ac.kr \\ http://mcl.yonsei.ac.kr
}

\begin{abstract}
Throughput performance and geographical service fairness of best effort service used for downlink of a 802.16e based TDD-OFDMA sectored cellular networks are evaluated in conjunction with different scheduling schemes and frequency reuse plans. The OFDM systems are based on two multiple access schemes, which are the OFDM-TDM and OFDMA, and considered scheduling schemes are round robin, max C/I, $\mathrm{PF}$ and G-fair schedulers with adaptive rate control. The 3 -sectored 1 FA, 3-sectored $3 \mathrm{FA}$, and 6-sectored $3 \mathrm{FA}$ plans are compared in terms of throughput, capacity, and geographical service fairness, which assist in determining the choice of a scheduling and frequency reuse plan.
\end{abstract}

\section{Introduction}

Link adaptation and channel scheduling are key techniques used in dynamic resource management for $3 \mathrm{G}$ wireless networks [1]. The single carrier $3 \mathrm{G}$ technologies, which are 1xEVolution data optimized (1xEV-DO) [2], 1xEVolution data and voice (1xEV-DV) [3] or high speed downlink packet access (HSDPA) [4], have proposed fast link adaptation as well as packet scheduling within the time domain. Multi-user time-domain schedulers have nearly doubled overall system capacity by handling fading channel time variation.

Emerging WLANs and future wireless mobile systems are expected to be based on a multi-carrier scheme (OFDM) with hundreds of carriers [1]. For a broadband channel, frequency variation as well as time variation should be handled more carefully in order to enhance overall performance. There are two strategies applied to combat frequency variations: "frequency diversity" and "frequency selectivity" [5]. With frequency diversity, the identical modulation and coding scheme is performed over all subchannels in order to prevent system performance from being dominated by a few deeply faded subcarries. In contrast, the frequency selective strategy can exploit frequency variations knowledge in order to provide link adaptation gains. The different signal quality values occurring at each subchannel can adopt different link adaptations and channel schedulers for use with each sub-band [5][6]. 
IEEE standards 802.16 [7] and 802.16a [8], which define the fixed broadband wireless access (FBWA) system, are based on OFDM/OFDMA transmission technologies. The IEEE 802.16 task group e (802.16 TGe) started in order ensure coexistence between fixed and mobile broadband wireless access (FMBWA) with fixed and mobile user terminals (UTs). The 802.16e amendment aims at the mobility support in 802.16 standard in 2 to $6 \mathrm{GHz}$ licensed bands [9]. In this paper, the $802.16 \mathrm{e}$ is considered for use in OFDMA cellular networks and the overall performance of packet scheduling is evaluated for specific frequency reuse plans by performing simulations.

The remainder of the paper is organized as follows. In Section 2, the basic system model for 802.16e based OFDMA cellular networks is introduced. In Section 3, downlink scheduling schemes are presented. The overall performance of the schemes is evaluated in Section 4 by simulation. Finally, conclusions are made in Section 5.

\section{OFDMA Cellular Networks}

\subsection{IEEE 802.16e PHY Frame Structure}

The 802.16e PHY is based on OFDM/OFDMA and is highly aligned with the 802.16a. Furthermore, time division duplexing (TDD) is considered as a duplexing method. Fig. 1 shows the 802.16e PHY frame structure. The fixed length OFDMA frame consists of successive downlink (DL) OFDM symbols followed by successive (UL) OFDM symbols. The Tx/Rx transition gap (TTG) is defined as the transition gap between DL and UL, and the Rx/Tx transition gap (RTG) is defined as the transition gap between UL and DL. In the frequency domain, full RF bandwidth is divided into hundreds of subcarriers. The number of subcarriers is equal to the OFDM FFT size. Numbers of subcarriers are bunched into the subchannel, which can be handled as a minimal resource unit. The subchannel size is assumed to be larger than the coherence bandwidth [8][9].

\subsection{OFDM-TDM Versus OFDMA}

IEEE 802.16 specifies two categories of OFDM systems: one is OFDM-TDM and the other is OFDMA [8][9]. In the first scheme, all carriers are simultaneously assigned for transmission and downstream data is time-division multiplexed (TDM). The OFDMA represents the time and frequency division multiple access technique based on the OFDM. A number of a specific UT's traffic can be transmitted simultaneously by using different subchannels. Subchannels are allocated dynamically within 802.16e. There are two dynamic subchannel allocation methods for multiple access usage, which include the media access protocol (MAP) and frequency hopping (FH). In MAP based OFDMA, the data streams are assigned on subchannels through MAP messages sent via downstream transmission. In this case, MAP can be scheduled dynamically by the base station (BS). In FH-OFDMA, the data streams are assigned on subchannels by using 


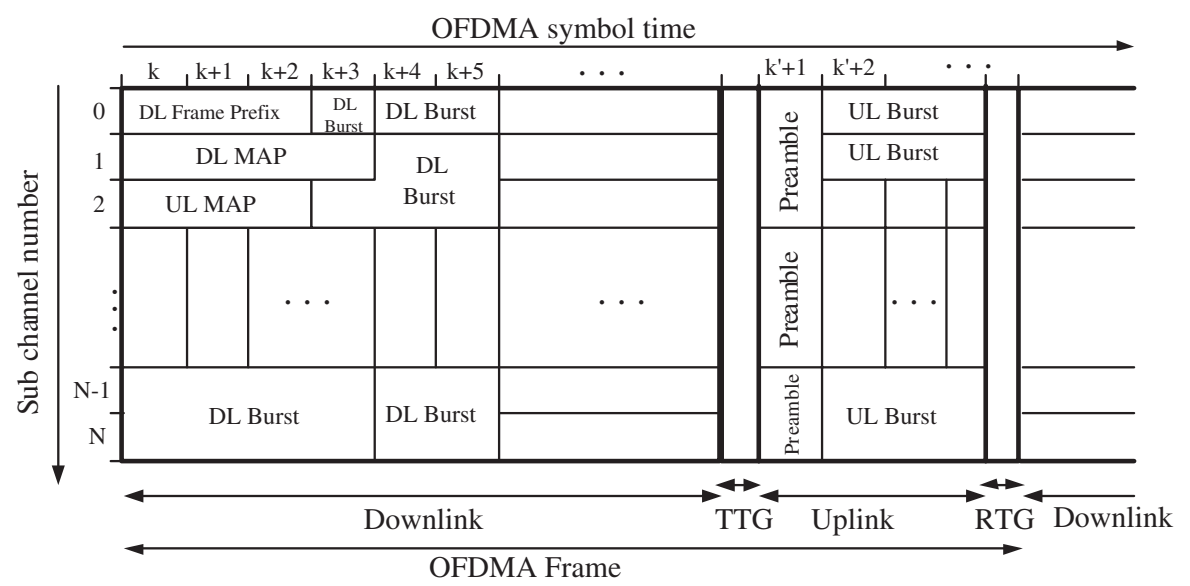

Fig. 1. IEEE 802.16e OFDMA frame structure

a hopping sequence, which is assigned to the UT during the call setup procedure. The FH-OFDMA technique is difficult to coordinate with dynamic resource management schemes such as packet scheduling or link adaptation.

\subsection{Single Frequency Network and Multi Frequency Network}

Two frequency reuse plans are considered: one is a single-frequency network (SFN) and the other is a multi-frequency network (MFN) [9]. Subsequently, multi-cell can be designed by single sectored or multi-sectored modes. The frequency reuse factor (FRF) is defined as the ratio between total number of cells (sectors) and the number of cells (sectors) that use the same frequency allocation (FA). Fig. 2 shows the examples of a frequency reuse plan: (a) shows SFN examples and (b) shows MFN examples. The "111" plan is a SFN with single sector cells and the "131" plan is a SFN with three-sector cells, in which the FRF equals 1. However, SFN experiences severe interference problems in the cell (sector) edge area. The "133" plan has 3-sectored cells in which each sector is operated at a different FA. In multi-frequency plans, edge area interference problems are improved, but frequency efficiency decreases.

\section{Downlink Scheduling for TDD-OFDMA Networks}

There are two types of scheduling: one is channel state independent scheduling and the other is channel state dependant scheduling. It is assumed that MAP is used to assign scheduled resources to UTs. These UTs measure the channel quality by using the received downlink pilot, and upload the proper modulation and coding scheme (MCS) levels to the BS through uplink feedback channels. Then, the BS schedules the downlink stream and transmits the UT's traffic by using the reported MCSs. 


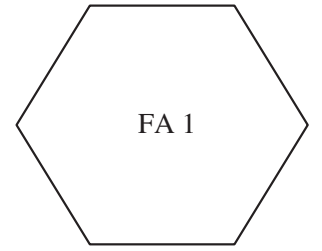

111

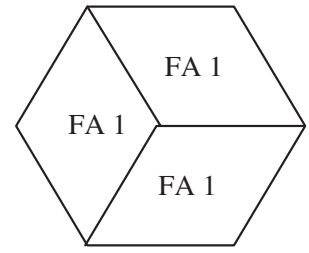

131

(a) Single frequency networks (1 FA is needed, FRF $=1$ )

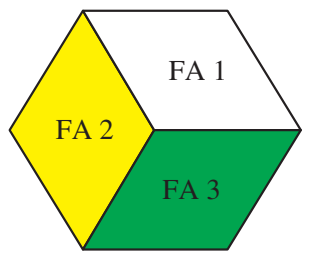

133

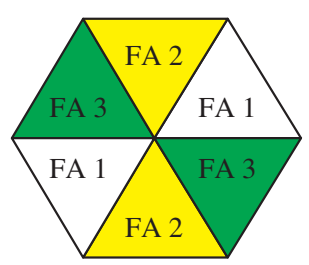

163

(b) Multi frequency networks (3 FAs are needed, FRF $=3$ )

Fig. 2. Frequency reuse plan examples (surrounding cells are not shown, but are repeatedly present)

\subsection{Round Robin (RR) Scheduling for TDD-OFDMA}

A round robin (RR) represents channel state independent scheduling and does not guarantee throughput fairness in a wireless condition [1]. Fig. 3 shows round robin scheduling examples: (a) is the TDD-OFDM/TDM downlink and (b) is TDD-OFDMA downlink. Channel information is not needed for RR scheduling, but needed for adaptive rate control. In the OFDM/TDM system, scheduling for OFDMA symbol by only time division manner would be feasible. The RR scheduler of a specific OFDMA should be able to perform in the cluster unit which is defined as the group of contiguous subcarriers and OFDMA symbols. The minimal cluster will be one subchannel occurring during one OFDMA symbol.

\subsection{Channel State Dependant Scheduling for TDD-OFDMA}

Since a channel scheduler should use the available channel information, the scheduling time unit is determined to be the channel feedback period. In a frequency division duplexing (FDD) system such as $1 \mathrm{xEV}$ or HSDPA, continuous downstream and upstream channels coexist. Therefore, the scheduling period can be converged to fit the minimal transmission slot duration, which is generally used during the channel feedback period. The time division duplexing (TDD) system does not provide continuous downstream nor continuous upstream. The channel feedback period is determined to be the OFDMA frame duration. Therefore, scheduling can be performed during every frame. Fig. 4 (a) shows the channel scheduling example occurring in TDD-OFDM/TDM. Since channel feedback is reported every frame, only one user can be allocated for one DL frame. How- 


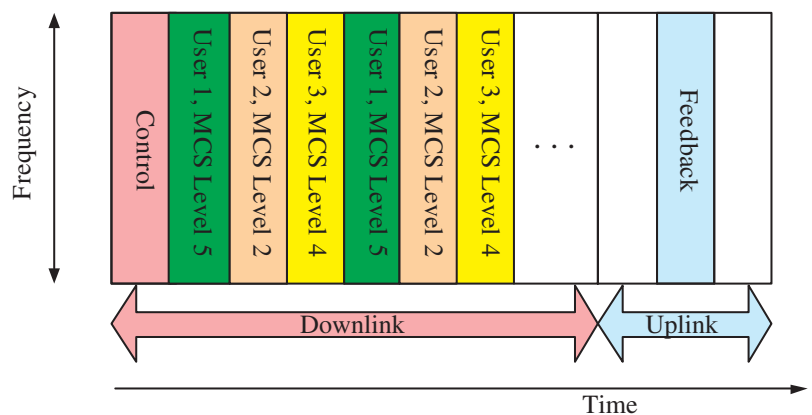

(a) Round robin scheduling in a TDD-OFDM/TDM downlink

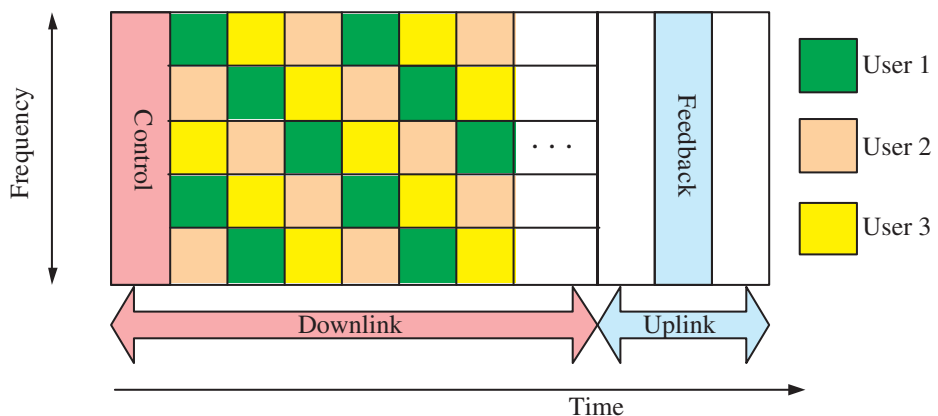

(b) Round robin scheduling in a TDD-OFDMA downlink

Fig. 3. Round Robin Scheduling Examples of TDD-OFDM/OFDMA Downlinks (adaptive rate control is performed, but power control is not performed)

ever, an OFDMA scheduler can allocate the frequency subchannels to different users as shown in Fig. 4 (b).

\subsection{Conventional Channel Schedulers}

One simple method is to serve the UT of index $i_{n}^{*}$ at the $n$-th subchannel for every scheduling instance $t$, with respect to:

$$
i_{n}^{*}=\arg \max _{i} M C S_{i, n}(t),
$$

where $M C S_{i, n}(t)$ denotes the MCS level of the $n$-th subchannel of the $i$-th UT. This scheduling resembles the "max C/I" scheduler, and will maximize the total downlink throughput of a base station. However, this high throughput is achieved at the cost of unfairness among the various UTs. To remedy this problem, it was suggested that the selected UT, $i_{n}^{*}$ should be denoted such that:

$$
i_{n}^{*}=\arg \max _{i} M C S_{i, n}(t) / T_{i, n}(t)
$$




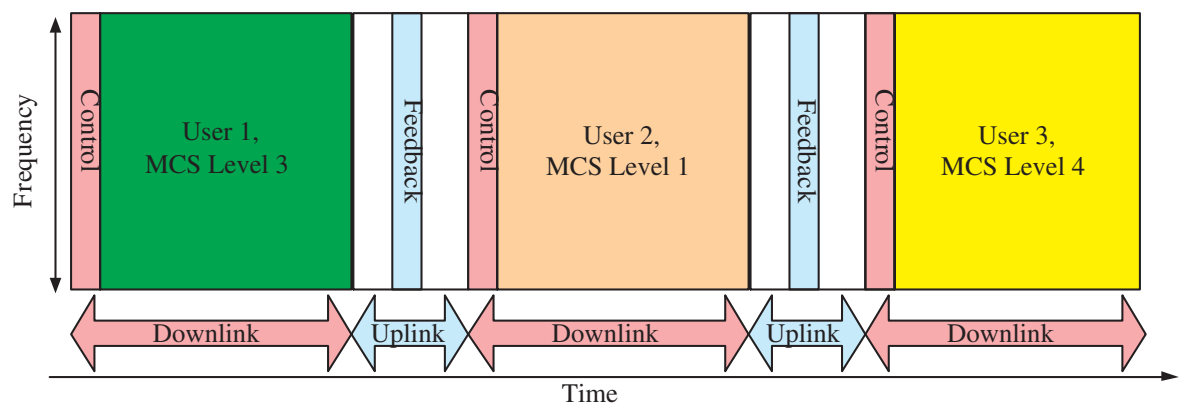

(a) Channel scheduling located within TDD-OFDM/TDM downlink

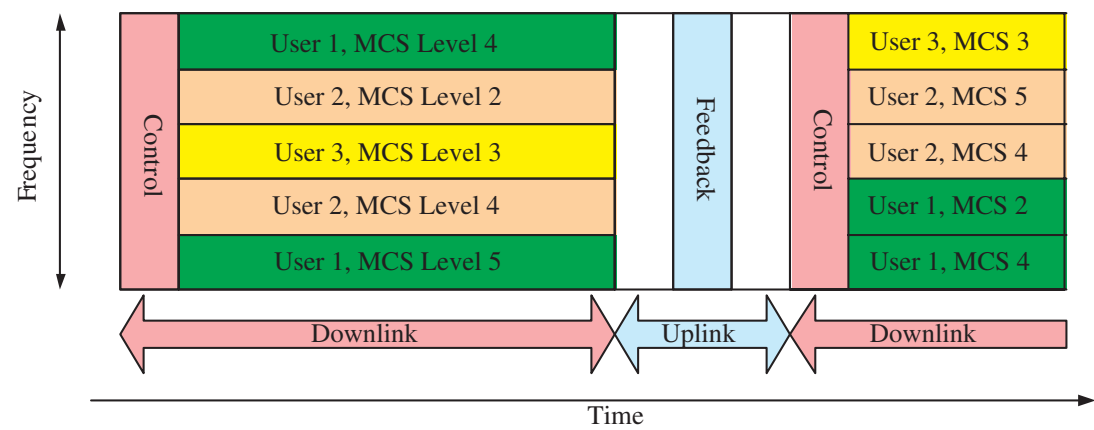

(b) Channel scheduling located within TDD-OFDMA downlink

Fig. 4. Priority-Based Channel Scheduling Examples used in TDD-OFDM/OFDMA Downlinks (adaptive rate control is performed, but power control is not performed)

where $T_{i, n}(t)$ denotes the average throughput of $n$-th subchannel of $i$-th UT. This scheme ensures proportional fairness (PF) scheduling algorithm [11]. In [12], a generalization of the proportional fair scheduler was shown, and it was suggested that the selected UT, $i_{n}^{*}$ should be defined that:

$$
i_{n}^{*}=\arg \max _{i} \frac{M C S_{i, n}(t)}{T_{i, n}(t)} \frac{h_{i}\left(M_{i, n}(t)\right)}{M_{i, n}(t)},
$$

where $M C S_{i, n}(t) / T_{i, n}(t)$ is a well-known $\mathrm{PF}$ factor and $M_{i, n}(t)$ is the average MCS. This scheduling is defined as G-fair scheduling algorithm. The expression $h(x)$ is a user-specific function that specifies overall fairness behavior [12].

\section{Simulations: Performance in a Multi-cell Environment}

\subsection{16e OFDMA Parameters}

The 802.16e based TDD-OFDM/OFDMA system is considered [8][9]. Table 1 shows the OFDMA parameters, and the link adaptation table (MCS table) is 
set to the parameters displayed in Table 2 [15]. The MCS level is reported according to $\mathrm{S} / \mathrm{N}$ sensitivity thresholds, and one frame delay is considered as MCS feedback. An incorrect MCS report can occur due to time-varying channel and feedback delay. Therefore, the SNR decision method has been adopted to simulations. If the received $\mathrm{C} / \mathrm{I}$ can satisfy the $\mathrm{S} / \mathrm{N}$ sensitivity, the traffic is correctly received, otherwise the traffic is lost.

Table 1. OFDMA parameters

\begin{tabular}{cc}
\hline Parameters & Value \\
\hline Carrier Frequency & $2.3 \mathrm{GHz}$ \\
Channel Bandwidth & $10 \mathrm{MHz}$ \\
Number of used subcarriers & 1,702 of 2,048 \\
Number of traffic subcarriers & 1,536 \\
Subcarrier spacing & $5.57617 \mathrm{kHz}$ \\
Number of subchannels & 32 \\
Number of subcarriers & 48 \\
Frame length & $5.0 \mathrm{msec}$ \\
Number of symbols per frame & 26 \\
Number of DL symbols & 18 \\
Number of UL symbols & 8 \\
Sum of RTG and TTG & $45.885 \mu \mathrm{sec}$ \\
OFDMA symbol time & $190.543 \mu \mathrm{sec}$ \\
Guard interval & $11.208 \mu \mathrm{sec}$ \\
Cyclic prefix & $1 / 16$ \\
\hline
\end{tabular}

Table 2. Modulation and coding (MCS) table [15]

\begin{tabular}{cccc}
\hline Modulation & Code rate & Sensitivity threshold S/N & PHY bit/sec/Hz \\
\hline QPSK & $1 / 2$ & $6.6 \mathrm{~dB}$ & 0.807 \\
16QAM & $1 / 2$ & $10.5 \mathrm{~dB}$ & 1.613 \\
64QAM & $2 / 3$ & $15.3 \mathrm{~dB}$ & 3.227 \\
64QAM & $3 / 4$ & $20.8 \mathrm{~dB}$ & 3.63 \\
\hline
\end{tabular}

\subsection{Simulation Environments}

It is assumed that there are 19 cells located within a $1 \mathrm{~km}$ radius. These 16 UTs are distributed in the center cell and the UT's location is generated more than 1,000 times. All UTs are assumed to be best-effort traffic with full-buffering. The channel $\mathrm{C} / \mathrm{I}$ of the $n$-th subchannel of $i$-th UT is assumed to be:

$$
(C / I)_{i, n}=\sum_{j=1}^{J}\left\|\gamma_{j, n}\right\|^{2} \cdot\left(G_{i}^{-1}+\sum_{k=1}^{K}\left\|\psi_{k, n}\right\|^{2}\right)^{-1}
$$


where $G_{i}$ is the average geometry which is determined by path loss and shadowing and shown as

$$
G_{i}=\frac{I_{o r}}{I_{o c}+N_{0} W}=\frac{1}{I_{o c} / I_{o r}+1 /\left(I_{o r} / N_{0} W\right)},
$$

where $I_{o r}$ is the received serving-cell pilot strength, $I_{o c}$ is the sum of the received other-cell pilot strength, and $N_{0} W$ is the thermal noise power. The expression $\left\{\gamma_{j}\right\}$ represents the multi-path component within the guard interval, but $\left\{\psi_{k}\right\}$ is multi-path component which exceeds the guard interval. In simulations,

$\sum_{j=1}^{J}\left\|\gamma_{j, n}\right\|^{2}$ is assumed to be Rayleigh fading and $\left\{\psi_{k}\right\}$ is ignored, since the cyclic prefix is assumed to be sufficiently larger than the overall delay spread. The path loss model is assumed to be a vehicular model $129.427+37.6 * \log _{10}\left(d_{k m}\right)$ [14]. The standard deviation of Log-normal shadowing is assumed to be $10 \mathrm{~dB}$. Short-term channel gains are assumed to be Rayleigh fading with a Doppler frequency of $6.4 \mathrm{~Hz}(3 \mathrm{~km} / \mathrm{Hr})$ and no correlation between subchannels is assumed. The BS transmit power is set to $20 \mathrm{~W}(43 \mathrm{dBm})$ and antenna gain is set to 14 $\mathrm{dBi}$. Thermal noise density is assumed to be $-174 \mathrm{dBm} / \mathrm{Hz}$ and max $\mathrm{C} / \mathrm{I}$ limit is set to $30 \mathrm{~dB}$.

Each Ring is defined as the area occupied by a 100 meter unit. For example, the $n$-th Ring is the area in that distance from the BS which are from $(n-1)$ hundred meters to $n$ hundred meters. Three frequency reuse plans are simulated: 131 ( 3 sector, 1 FA), 133 (3 sector, 3 FA), and 163 (6 sector 3 FA). Fig. 5 shows the average geometry distribution obtained by simulations. The 133 plan shows the best received geometry over almost all rings among the simulated frequency reuse plans, but it should be noted that the 133 plan has three FRF.

\subsection{Throughput Performance}

The four scheduling schemes, which include RR, max C/I, PF, and G-fair with $h(x)=1$. Table 3 shows overall sector throughput. Sector throughput of the max $\mathrm{C} / \mathrm{I}$ scheduler displays maximum sector capacity. In the OFDM/TDM system, throughput of the PF is close to that of the RR, but throughput of the PF is around 2 to 3 times higher than that of the RR for the OFDMA system. Furthermore, throughput of the G-fair is around half of that of the RR in OFDM/TDM, but throughput of the G-fair in the OFDMA is 1.5 to 2.5 times higher than that of the RR. Since the RR is performed during the symbol period while channel schedulers are working during every frame period, the TDM system produces lower multi-user scheduling gain. However, the OFDMA produces subsequently increased multi-user scheduling gain by utilizing frequency domain scheduling. For RR and max C/I, the throughput difference between TDM and OFDMA is negligible, but OFDMA throughput is around 2 to 3 times higher than that of the TDM in terms of PF and G-fair.

Table 4 shows normalized throughput determined as

$$
T_{\text {Normalized }}=\frac{T_{\text {Sector }} \cdot N_{\text {Sector }}}{B W \cdot F R F},
$$




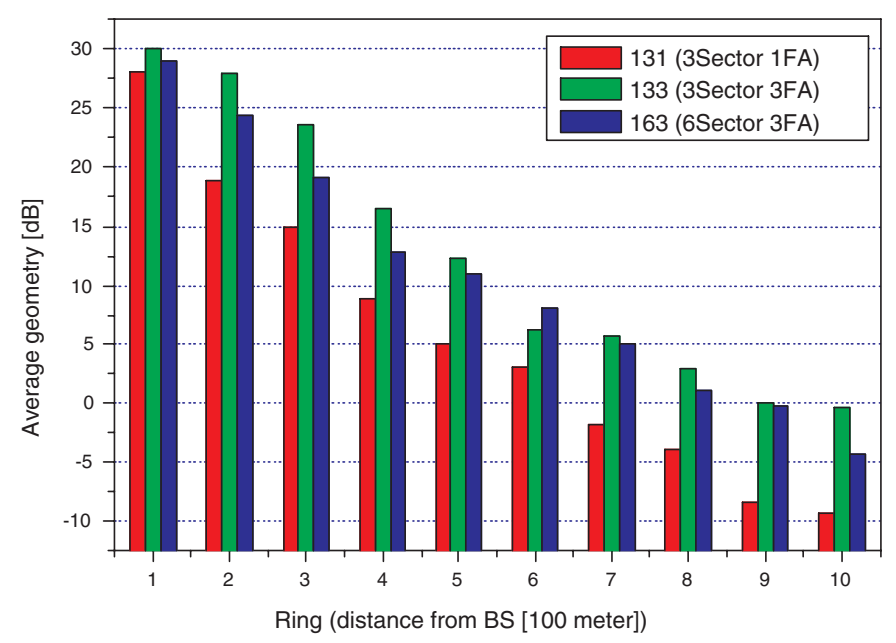

Fig. 5. Average received geometry for different frequency reuse plans

Table 3. Sector throughput [kbps/Sector]

\begin{tabular}{ccccccc}
\hline & \multicolumn{2}{c}{$131(3$ sector 1FA $)$} & \multicolumn{2}{c}{$133(3$ sector 3FA $)$} & \multicolumn{2}{c}{$163(6$ sector 3FA $)$} \\
\cline { 2 - 7 } & TDM & OFDMA & TDM & OFDMA & TDM & OFDMA \\
\hline RR & $2,279.14$ & $2,193.67$ & $4,813.04$ & $4,669.42$ & $4,224.99$ & $4,042.41$ \\
Max C/I & $12,019.15$ & $12,179.38$ & $16,333.79$ & $16,478.59$ & $14,630.79$ & $14,783.21$ \\
PF & $2,301.14$ & $7,000.82$ & $4,781.71$ & $9,361.92$ & $4,143.76$ & $8,452.39$ \\
G-Fair & $1,112.00$ & $5,343.32$ & $2,184.87$ & $6,743.79$ & $1,986.67$ & $5,804.66$ \\
\hline
\end{tabular}

where $T_{\text {Sector }}$ is sector throughput, $N_{\text {Sector }}$ is number of sectors per cell, and $B W$ is the channel bandwidth for each FA. Table 4 shows that normalized throughput of plan 131 is the highest and the 133 plan produces the smallest throughput calculated among the investigated plans. In other words, the 133 plan can reduce other-cell interference and increase sector throughput but has lower frequency efficiency. The 131 plan displays the lowest sector throughput, but has the largest normalized throughput.

The throughput of UTs placed at around cell edge is an important key necessary to understand cell coverage. Fig. 6 shows the average user throughput of the first Ring (best channel condition) and Fig. 7 shows the average user throughput of the 10-th Ring (worst channel condition). PF and G-fair schedulers can provide higher throughput than $\max \mathrm{C} / \mathrm{I}$ in worst channel condition users. The PF and G-fair scheduler can provide more than $200 \mathrm{kbps}$ to 10-th Ring users in the 133 plan, and can provide around $150 \mathrm{kbps}$ in the 163 plan. Average user throughput of the 10-th Ring observed in the 131 plan is less than 
Table 4. Normalized throughput [bps/Hz/Cell]

\begin{tabular}{ccccccc}
\hline & $131(3$ sector 1FA $)$ & \multicolumn{2}{c}{$133(3$ sector 3FA $)$} & $163(6$ sector 3FA $)$ \\
\cline { 2 - 7 } & TDM & OFDMA & TDM & OFDMA & TDM & OFDMA \\
\hline RR & 0.68 & 0.66 & 0.48 & 0.47 & 0.84 & 0.81 \\
Max C/I & 3.61 & 3.65 & 1.63 & 1.65 & 2.93 & 2.96 \\
PF & 0.69 & 2.10 & 0.48 & 0.94 & 0.83 & 1.69 \\
G-Fair & 0.33 & 1.60 & 0.22 & 0.67 & 0.40 & 1.16 \\
\hline
\end{tabular}

80 kbps. Therefore, though the 131 plan provides the highest channel capacity, it is not suitable for service in the cell edge area. Fig. 8 shows the average user throughput for each PF scheduling Ring for OFDMA schemes. It is shown that while the OFDMA 131 plan PF scheduler provides $70 \mathrm{kbps}$ to $3.6 \mathrm{Mbps}$ of user throughput, the 133 plan PF scheduler can provide $240 \mathrm{kbps}$ to $2.4 \mathrm{Mbps}$ and the 163 plan can provide $150 \mathrm{kbps}$ to $2.1 \mathrm{Mbps}$.

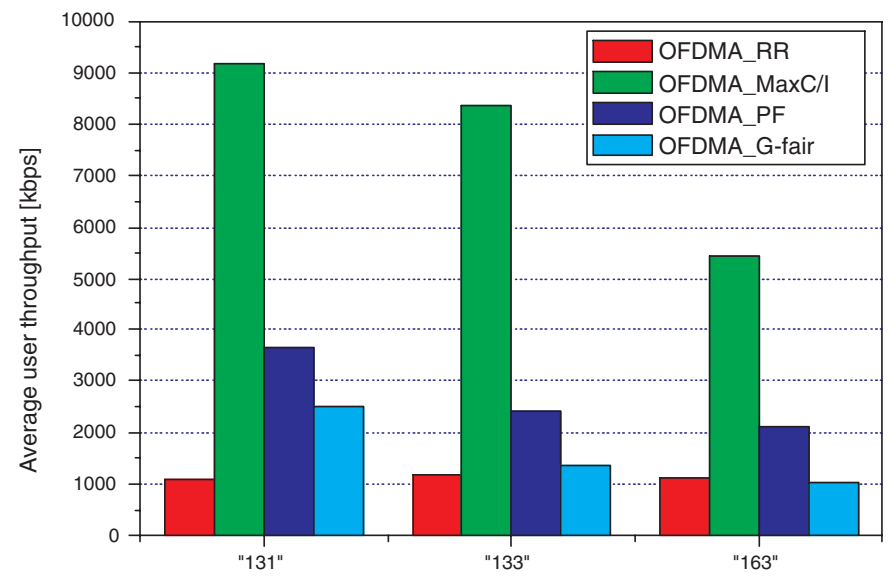

Fig. 6. Average user throughput in the first Ring (distance from BS: 0 to 100 meters) for the OFDMA system ("131": 3 sector 1 FA, "133": 3 sector 3 FA, "163": 6 sector 3 FA)

\subsection{Fairness Performance}

Figs 9 to 11 show the fairness CDF curves for use in OFDMA scheduling schemes for the 131, 133, and 163 plans. Fairness is measured by the throughput to average throughput ratio. The spike located at zero on the $x$-axis is a result of poor channel conditions or scheduling starvation. The max C/I scheduler 


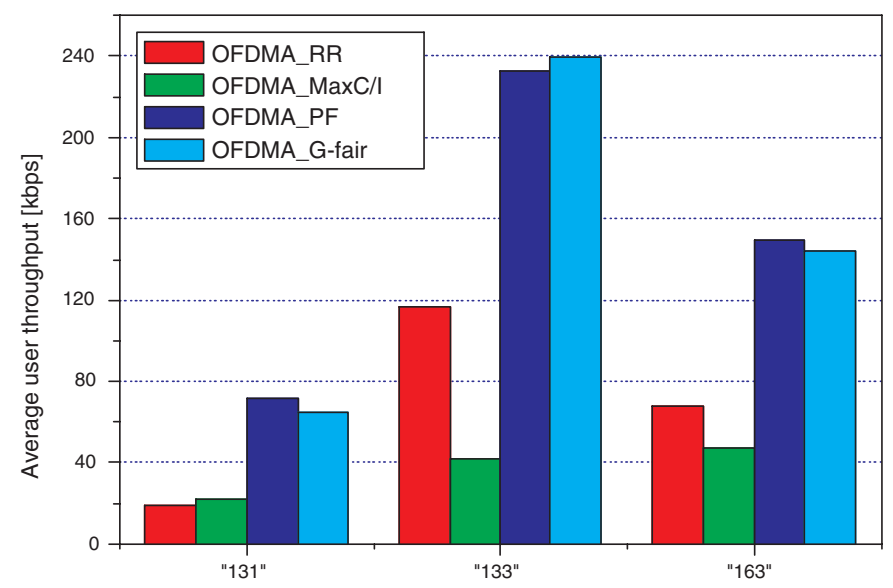

Fig. 7. Average user throughput in the 10-th Ring (distance from BS: 900 to 1,000 meters) for the OFDMA system ("131": 3 sector 1 FA, "133": 3 sector 3 FA, "163": 6 sector $3 \mathrm{FA}$ )

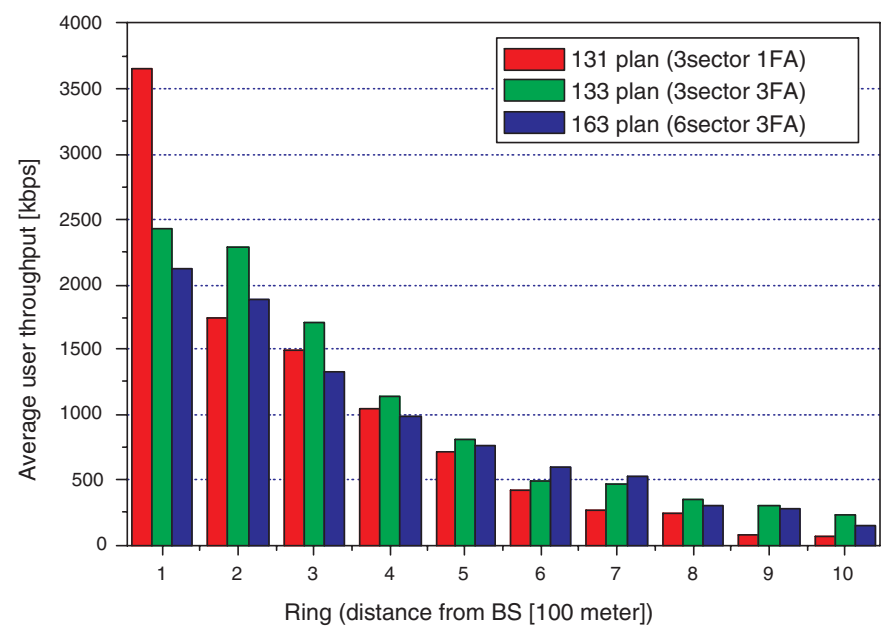

Fig. 8. Average user throughput of PF schedulers for use in an OFDMA system

accounts for approximately $90 \%$ of the faded UTs that could not be served. Other schedulers account for around $65 \%, 50 \%$, and $50 \%$ fade for 131,133 , and 163 plans respectively. Since the max C/I serves only the best channel condition UT, it displays severe unfairness for all investigated plans. As expected, the G- 
fair scheduler provides the best fairness. The 133 and 163 plans also show more fairness than the 131 plan for RR, PF and G-fair schedulers.

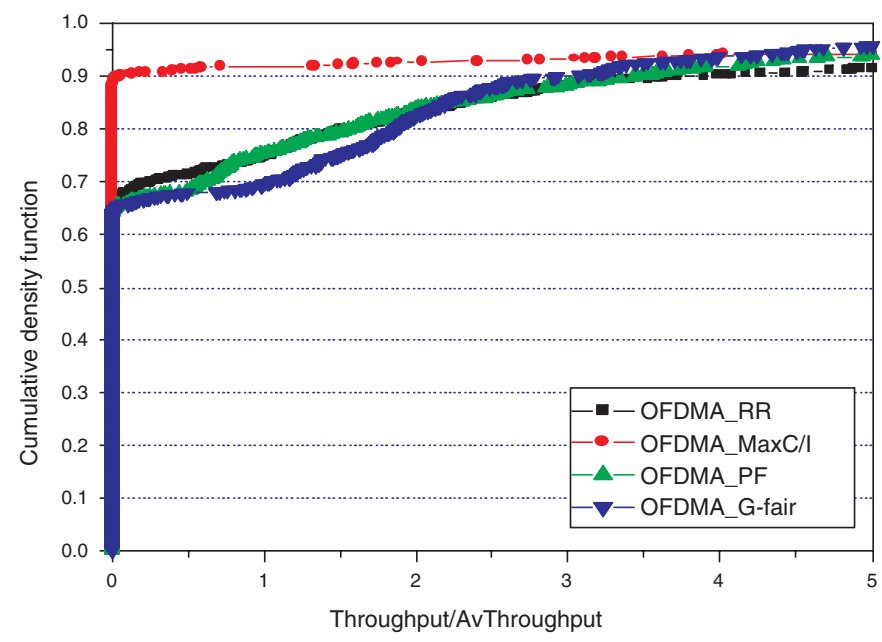

Fig. 9. Fairness curves for OFDMA scheduling schemes for use in the 131 plan (3 sector $1 \mathrm{FA})$

\section{Concluding Remarks}

Throughput and its geographical service fairness of best effort service used for downlink of 802.16e based TDD-OFDMA sectored cellular networks were evaluated in conjunction with different scheduling schemes and frequency reuse plans. Both OFDM/TDM and OFDMA was considered and the 131 (3-sectored 1 FA), 133 (3-sectored $3 \mathrm{FA}$ ) and 163 (6-sectored $3 \mathrm{FA}$ ) plans were considered for use in a frequency reuse plan. The round robin, $\max \mathrm{C} / \mathrm{I}, \mathrm{PF}$ and G-fair schedulers were evaluated along with adaptive rate control. The 133 plan shows the best throughput and geographical service fairness among the investigated plans, while it produces the worst frequency efficiency. Results indicate that the choice of a scheduling and frequency reuse plan should be determined with consideration in terms of the trade-off between system throughput and geographical service fairness. 


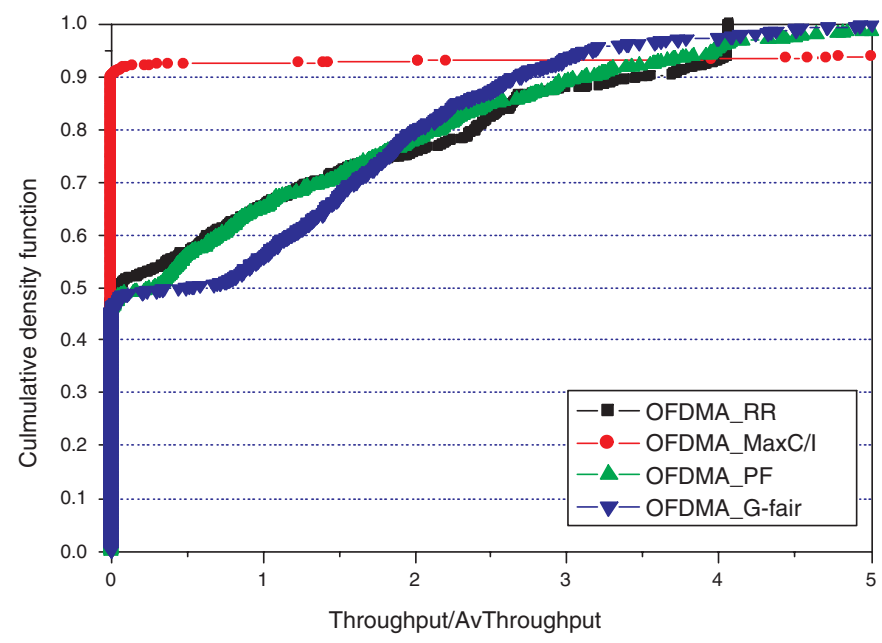

Fig. 10. Fairness curves for OFDMA scheduling schemes for use in the 133 plan (3 sector $3 \mathrm{FA}$ )

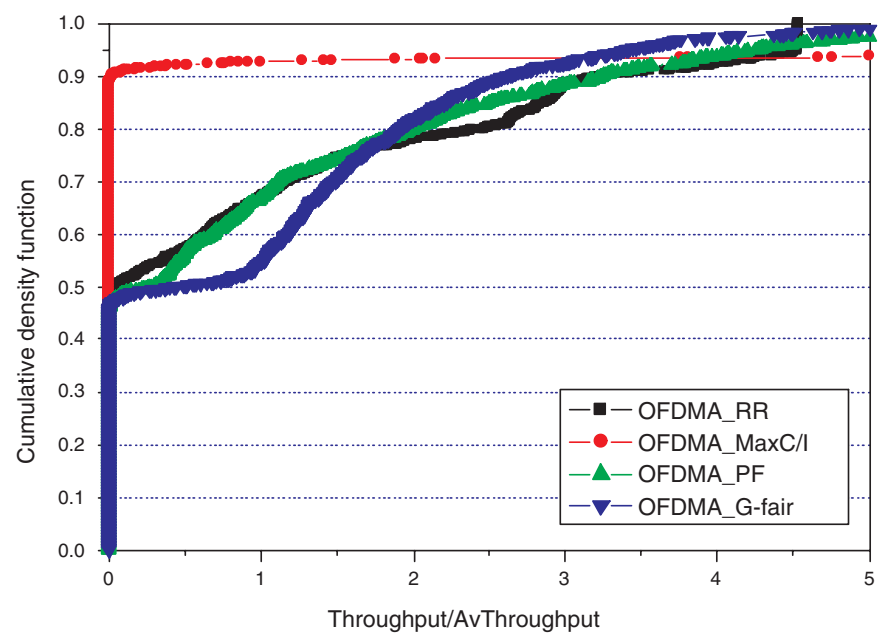

Fig. 11. Fairness curves for OFDMA scheduling schemes for use in the 163 plan (6 sector $3 \mathrm{FA}$ )

Acknowledgments. This work was supported by grant No. (R01-2002-00000531-0) from the Basic Research Program of the Korea Science \& Engineering Foundation. Also, the work was supported by Qualcomm Incorporated through Qualcomm Yonsei CDMA Joint Research Center. 


\section{References}

1. Shakkottai S., Rappaport T. S.: Cross-Layer Design for Wireless Networks. Communications Magazine, Vol. 41. IEEE. (2003) 74-80

2. TIA/EIA/IS-856. cdma2000 High Rate Packet Data Air Interface Specification, (2001)

3. cdma2000 release C. C.S000X-C, (2002)

4. 3GPP release 5. TS-25.211-25.214, (2002)

5. Classon B., Sartori P., Nangia V., Zhuang X., Baum K.: Multi-dimensional Adaptation and Multi-user Scheduling Techniques for Wireless OFDM Systems. IEEE International Conference on Communications (ICC '03), (2003)

6. Anchun W., Liang X., Shidong Z., Xibin X., Yan Y.: Dynamic Resource Management in the Forth Generation Wireless Systems. International Conference on Communication Technology (ICCT2003), (2003)

7. IEEE Standard 802.16, IEEE Standard for Local and Metropolitan Area Networks Part 16: Air Interface for Fixed Broadband Wireless Access Systems, (2001)

8. IEEE Standard 802.16a, IEEE Standard for Local and Metropolitan Area Networks Part 16: Air Interface for Fixed Broadband Wireless Access Systems Amendment2: Medium Access Control Modification and Additional Physical Layer Specifications for $2-11 \mathrm{GHz},(2003)$

9. http://www.ieee802.org/16/tge/

10. Nee R., Prasad R.: OFDM for Wireless Multimedia Communications. Artech House Publishers. (2000)

11. Bender P., Black P., Grob M., Padovani M., Sindhushayana N., Viterbi A.: CDMA/HDR: A Bandwidth-Efficient High-Speed Wireless Data Service for Nomadic Users. Communications Magazine, Vol. 38. IEEE. (2000) 70-77

12. Application Note: G-fair Scheduler. 80-H0551-1 Rev. B. Qualcomm Incorporated. (2002)

13. S. Keshav: An Engineering Approach to Computer Networking: ATM Networks, the Internet, and the Telephone Network. Addison Wesley. (1997)

14. Recommendation ITU-R M.1225, Guideline for Evaluation of Radio Transmission Technologies for IMT-2000, (1997)

15. IEEE C802.16e-03/22r1, Convergence simulations for OFDMA PHY mode, (2003) 